University of New Orleans

ScholarWorks@UNO

2-15-1985

\title{
Single-layer antireflection coatings on absorbing substrates for the parallel and perpendicular polarizations at oblique incidence
}

R. M.A. Azzam

University of New Orleans, razzam@uno.edu

Follow this and additional works at: https://scholarworks.uno.edu/ee_facpubs

Part of the Electrical and Electronics Commons, and the Optics Commons

\section{Recommended Citation}

R. M. A. Azzam, "Single-layer antireflection coatings on absorbing substrates for the parallel and perpendicular polarizations at oblique incidence," Appl. Opt. 24, 513-518 (1985)

This Article is brought to you for free and open access by the Department of Electrical Engineering at ScholarWorks@UNO. It has been accepted for inclusion in Electrical Engineering Faculty Publications by an authorized administrator of ScholarWorks@UNO. For more information, please contact scholarworks@uno.edu. 


\title{
Single-layer antireflection coatings on absorbing substrates for the parallel and perpendicular polarizations at oblique incidence
}

\author{
R. M. A. Azzam
}

\begin{abstract}
Explicit equations are derived that determine the refractive index of a single layer that suppresses the reflection of $p$ - or $s$-polarized light from the planar interface between a transparent and an absorbing medium at any given angle of incidence. The required layer thickness and the system reflectance for the orthogonal unextinguished polarization also follow explicitly. This generalizes earlier work that was limited to normal incidence or to oblique incidence at dielectric-dielectric interfaces. Specific examples are given of $p$ - and $s$-antireflection layers on $\mathrm{Si}$ and $\mathrm{Al}$ substrates at $\lambda=6328 \AA$ at various angles of incidence.
\end{abstract}

\section{Introduction}

Single-layer antireflection coatings on dielectric substrates for normally incident monochromatic light are well known. ${ }^{1}$ The layer refractive index $N_{1}$ must be chosen as ${ }^{2}$

$$
N_{1}=\left(N_{0} N_{2}\right)^{1 / 2},
$$

i.e., equal to the geometric mean of the refractive indices $N_{0}$ of the ambient (incidence medium) and $N_{2}$ of the substrate.

At a general angle of (oblique) incidence $\phi$, the condition of zero reflection by a transparent film on a transparent substrate, for the parallel $p$ or perpendicular $s$ polarization, has also been derived in explicit form, ${ }^{3}$ yielding $N_{1}$ as a function of $N_{0}, N_{2}$, and $\phi$.

When the substrate is absorbing, antireflection at normal incidence continues to be possible using a transparent film of refractive index ${ }^{4}$

$$
N_{1}=\left(N_{0} n_{2}+\frac{N_{0}^{2} k_{2}^{2}}{N_{0} n_{2}-N_{0}^{2}}\right)^{1 / 2},
$$

where $N_{2}=n_{2}-j k_{2}$ is the substrate complex refractive index. Equation (2) reduces to Eq. (1) when $k_{2}=0$.

In this paper we further generalize these earlier results and derive explicit equations for the refractive index of a transparent film on an absorbing substrate

The author is with University of New Orleans, Department of Electrical Engineering, Lakefront, New Orleans, Louisiana 70148.

Received 10 September 1984.

0003-6935/85/040513-06\$02.00/0.

(C) 1985 Optical Society of America. necessary to suppress the reflection of $p$ - or $s$-polarized light at any given angle of incidence. The thickness of the antireflection (polarizing) layer, and the associated unextinguished reflectance (for the orthogonal polarization) of the film-substrate system, are also determined. The results are illustrated by specific examples of antireflection layers on semiconducting (Si) and metallic (Al) substrates at one wavelength $(\lambda=6328$ $\AA$ ).

\section{Basic Relations}

In what follows, we will consider the antireflection condition for $p$-and $s$-polarized light separately. For either polarization, zero reflection by the ambientfilm-substrate (0-1-2) system happens if the ambient-film and film-substrate interface reflectances are equal:

$$
\left|r_{01 \nu}\right|=\left|r_{12 \nu}\right|, \nu=p, s .
$$

Equation (3) is basic and has been recognized previously. ${ }^{5-7}$ However, it appears that no attempt has been made to solve it for the film refractive index $N_{1}$ when the substrate is absorbing. ${ }^{8}$ With $N_{2}$ complex $r_{12 \nu}$ is also complex. In this case, manipulating Eq. (3) is simplified considerably by replacing it by the equivalent form

$$
r_{01 \nu}^{2}=r_{12 \nu} r_{12 \nu}^{*}
$$

where * indicates the complex conjugate.

Once $N_{1}$ that satisfies Eq. (3) or (4) has been determined, the normalized polarizing film thickness is readily obtained as ${ }^{6}$

$$
\zeta_{\nu}=-\arg \left(X_{\nu}\right) / 2 \pi, \quad 0 \leq \zeta_{\nu}<1,
$$

where 


$$
X_{\nu}=-r_{01 \nu} / r_{12 \nu}
$$

The least film thickness is

$$
d_{\nu}=\zeta_{\nu} D_{\phi},
$$

where

$$
D_{\phi}=\frac{\lambda}{2}\left(N_{1}^{2}-N_{0}^{2} \sin ^{2} \phi\right)^{-1 / 2}
$$

is the film thickness period and $\lambda$ is the free-space wavelength of light. Higher polarizing film thicknesses are obtained by adding integral multiples of $D_{\phi}$ to $d_{\nu}$.

The complex-amplitude reflection coefficient of the coated substrate for the unextinguished orthogonal polarization $\nu^{\prime}$ (if $\nu=p, \nu^{\prime}=s$, and vice versa) is

$$
R_{\nu^{\prime}}=\left(r_{01 \nu^{\prime}}+r_{12 \nu^{\prime}} X_{\nu}\right) /\left(1+r_{01 \nu^{\prime}} r_{12 \nu^{\prime}} X_{\nu}\right),
$$

where $X_{\nu}$ is given by Eq. (6). The corresponding intensity reflectance is

$$
\mathcal{R}_{\nu^{\prime}}=\left|R_{\nu^{\prime}}\right|^{2} .
$$

\section{Antireflection of the $s$ Polarization}

Fresnel's reflection coefficients of the ambient-film (01) and film-substrate (12) interfaces for the $s$ polarization are given by ${ }^{9}$

$$
\begin{aligned}
& r_{01 s}=\left(S_{0}-S_{1}\right) /\left(S_{0}+S_{1}\right), \\
& r_{12 s}=\left(S_{1}-S_{2}\right) /\left(S_{1}+S_{2}\right),
\end{aligned}
$$

where

$$
\begin{aligned}
S_{i} & =\left(\epsilon_{i}-\epsilon_{0} \sin ^{2} \phi\right)^{1 / 2}, \\
\epsilon_{i} & =N_{i}^{2}, i=0,1,2 .
\end{aligned}
$$

$\epsilon_{i}$ is the dielectric constant of medium $i$. On substituting Eqs. (11) into Eq. (4), the antireflection condition for $s$-polarized light takes the form

$$
\left(S_{0}^{2}+S_{1}^{2}\right) \operatorname{Re}\left(S_{2}\right)=S_{0}\left(S_{1}^{2}+\left|S_{2}\right|^{2}\right),
$$

where Re indicates the "real part of." To simplify reaching Eq. (14), we used the algebraic fact that if $(A$ $-B) /(A+B)=(C-D) /(C+D)$, then $A / B=C / D$. From Eq. (14) we get

$$
S_{1}^{2}=S_{0}\left[\left|S_{2}\right|^{2}-S_{0} \operatorname{Re}\left(S_{2}\right)\right] /\left[\operatorname{Re}\left(S_{2}\right)-S_{0}\right]
$$

If $S_{i}$ from Eq. (12) are used in Eq. (15), we obtain

$$
\begin{aligned}
& \epsilon_{1}=\epsilon_{0}\left\{\sin ^{2} \phi+\cos \phi\left[\frac{\left|\beta_{2}\right|-\cos \phi \operatorname{Re}\left(\beta_{2}{ }^{1 / 2}\right)}{\operatorname{Re}\left(\beta_{2}^{1 / 2}\right)-\cos \phi}\right]\right\}, \\
& \beta_{2}=\left(\epsilon_{2} / \epsilon_{0}\right)-\sin ^{2} \phi .
\end{aligned}
$$

Equation (16) gives the desired dielectric constant of the $s$-polarization antireflection layer in terms of the ambient dielectric constant $\epsilon_{0}$, substrate complex dielectric constant $\epsilon_{2}=\left(n_{2}-j k_{2}\right)^{2}$, and angle of incidence $\phi$. The corresponding refractive index is

$$
N_{1}=\epsilon_{1}^{1 / 2} .
$$

Knittl's result ${ }^{3}$ for a transparent film on a transparent substrate is obtained as a special case of Eq. (16) if the imaginary part of $\epsilon_{2}$ is set equal to zero.

As a first example, consider the reflection of light $(\lambda$ $=6328 \AA)$ in air $\left(\epsilon_{0}=1\right)$ by a Si substrate of complex

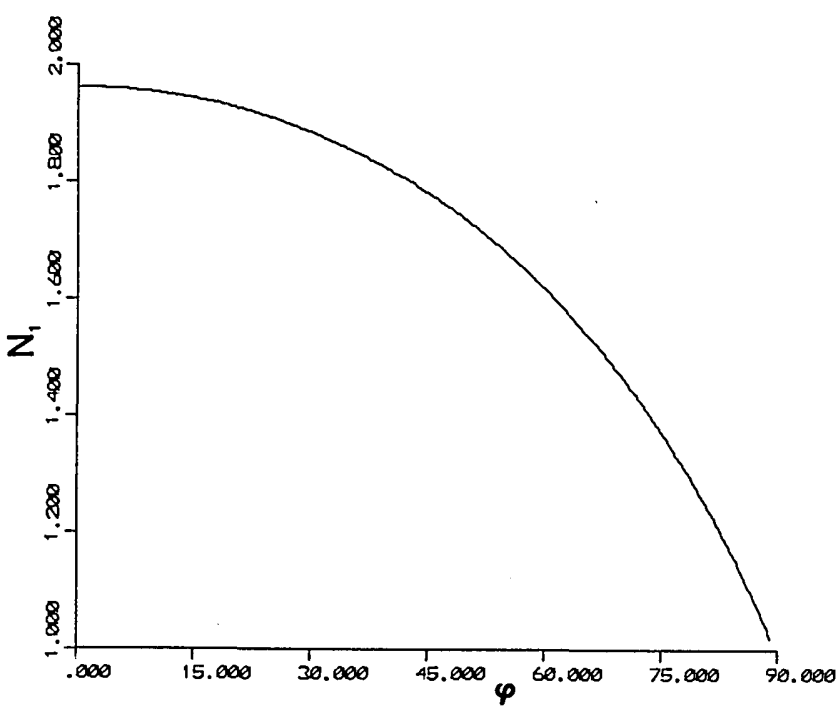

Fig. 1. Refractive index $N_{1}$ of $s$-polarization antireflection layer on $\mathrm{Si}\left(N_{2}=3.85-j 0.02\right)$ as a function of angle of incidence $\phi$ (degrees).

Light $(\lambda=6328 \AA)$ is assumed to be incident from air $\left(N_{0}=1\right)$.

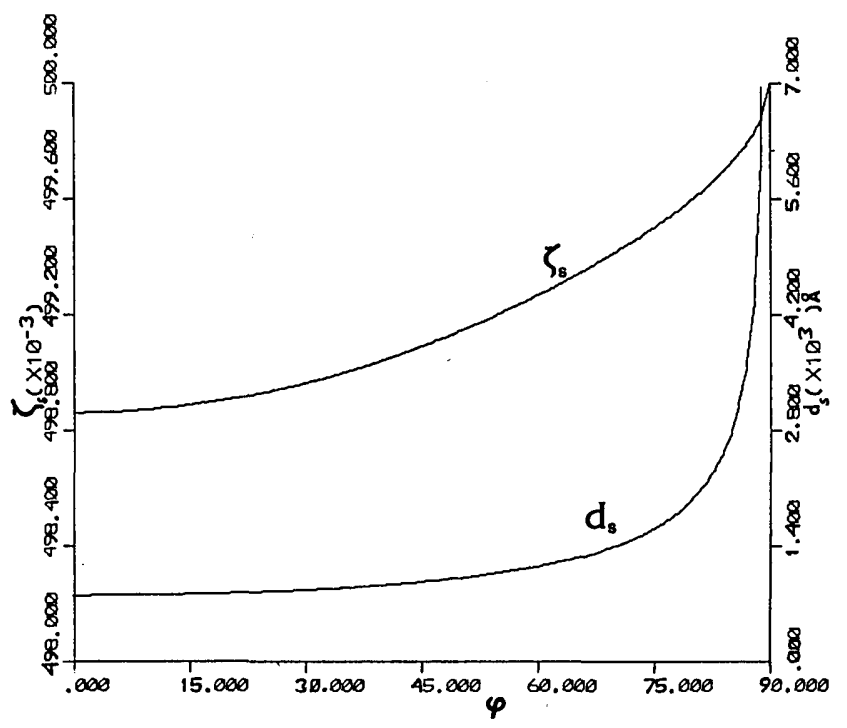

Fig. 2. Normalized $\zeta_{s}$ and actual $d_{s}$ (angstroms) thicknesses of the $s$-polarization antireflection layer on $\mathrm{Si}$ at $\lambda=6328 \AA$ vs angle of incidence $\phi$ (degrees).

refractive index ${ }^{10} N_{2}=3.85-j 0.02$. The refractive index $N_{1}$ of the $s$-polarization antireflection layer was computed from Eqs. (16)-(18) and is plotted in Fig. 1 as a function of $\phi$ from normal $(\phi=0)$ to grazing $(\phi=$ $90^{\circ}$ ) incidence. For $0 \leq \phi \leq 80^{\circ}$, we have $1.9622 \geq N_{1}$ $\geq 1.2713$, which correspond to several exisitng thin-film coating materials. ${ }^{11,12}$ For $\phi>80^{\circ}, N_{1}$ is too close to 1 to be realizable by a thin solid film.

Figure 2 shows the normalized and actual film thicknesses $\zeta_{s}$ and $d_{s}$, respectively, of the antireflection layer calculated from Eqs. (5)-(7). Because of the small but nonzero extinction coefficient of $\mathrm{Si}(0.02), \zeta_{s}$ is slightly less than one-half, and the layer is strictly not of quarterwave thickness. $\left(\zeta_{s}=1 / 2\right.$ holds exactly when the substrate is transparent and $k_{2}=0$.) 


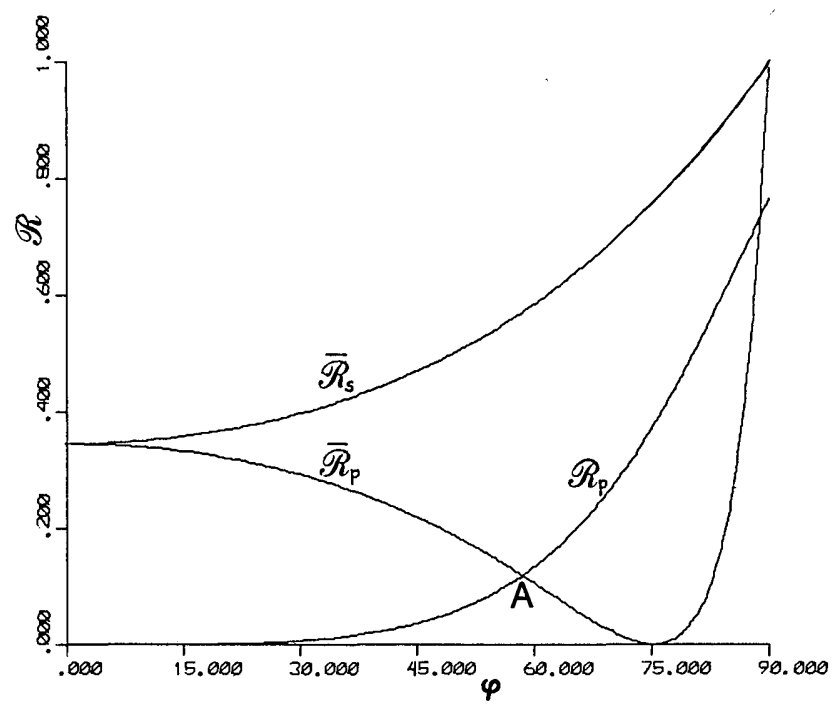

Fig. 3. Unextinguished $p$ reflectance $\mathscr{R}_{p}$ vs angle of incidence $\phi$ (degrees) for $\mathrm{Si}$, which is antireflection-coated for the $s$ polarization. $\overline{\mathscr{R}}_{p}$ and $\overline{\mathscr{R}}_{s}$ are the $p$ and $s$ reflectances of the bare Si substrate.

Table I. s-Polarization Antireflection Layers on Si at Five Angles of Incidence ${ }^{a}$

\begin{tabular}{cccrccc}
\hline$\phi$ & $N_{1}$ & $\zeta_{s}$ & \multicolumn{1}{c}{$d_{s}$} & \multicolumn{1}{c}{$\mathcal{R}_{p}$} & \multicolumn{1}{c}{$\bar{R}_{p}$} & $\bar{R}_{s}$ \\
\hline 0 & 1.96218 & 0.49886 & 804.4 & 0 & 0.3453 & 0.3453 \\
30 & 1.88576 & 0.49896 & 868.3 & 0.0063 & 0.2933 & 0.3971 \\
45 & 1.78218 & 0.49909 & 965.3 & 0.0361 & 0.2204 & 0.4694 \\
60 & 1.62041 & 0.49927 & 1153.4 & 0.1333 & 0.1075 & 0.5849 \\
75 & 1.37754 & 0.49950 & 1609.1 & 0.3710 & 0.0002 & 0.7571 \\
\hline
\end{tabular}

${ }^{a} \phi$ is the angle of incidence in degrees. $N_{1}$ is the layer required refractive index for $s$-polarization antireflection, and $\zeta_{s}$ is its normalized thickness (as a fraction of the thickness period). $d_{s}$ is the corresponding actual (least) thickness in angstroms. $\mathscr{R}_{p}$ is the reflectance of the coated Si for the unextinguished $p$ polarization. $\overline{\mathscr{R}}_{p}$ and $\overline{\mathcal{R}}_{s}$ are the reflectances of the film-free air-Si interface for the $p$ and $s$ polarizations, respectively. The complex refractive index of $\mathrm{Si}$ is taken as $N_{2}=3.85-j 0.02$ at $\lambda=6328 \AA$.

Figure 3 presents the unextinguished $p$ reflectance $\mathcal{R}_{p}$ of the coated $\mathrm{Si}$, computed from Eqs. (6), (9) and (10), plotted vs $\phi$. We have superimposed on Fig. 3 the reflectances $\overline{\mathcal{R}}_{p}$ and $\overline{\mathcal{R}}_{s}$ of the film-free air-Si interface. At point $A\left(\phi_{A}\right.$ is between 58 and $\left.59^{\circ}\right)$ Abelès's condition $^{13}$ of equal $p$ reflectances of the coated and uncoated substrate is satisfied. For $0 \leq \phi<\phi_{A}$, the coating that reduces the $s$ reflectance to zero also diminishes the $p$ reflectance below its bare-substrate value. At $\phi=0$, the $p$ and $s$ polarizations are indistinguishable, and total antireflection of light occurs. For $0<\phi \leq 45^{\circ}$, suppression of the $s$ polarization is accompanied by significant reduction of the $p$ reflectance, so that excellent (but incomplete) overall antireflection is achieved by one layer.

Table I lists data on $s$-polarization antireflection layers on $\mathrm{Si}$ (at $\lambda=6328 \AA$ ) at five angles of incidence $0,30,45,60$, and $75^{\circ}$. Silicon nitride is a particularly suitable antireflection coating material for any $\phi$ from 0 to $45^{\circ}$. If prepared by sputtering, its refractive index

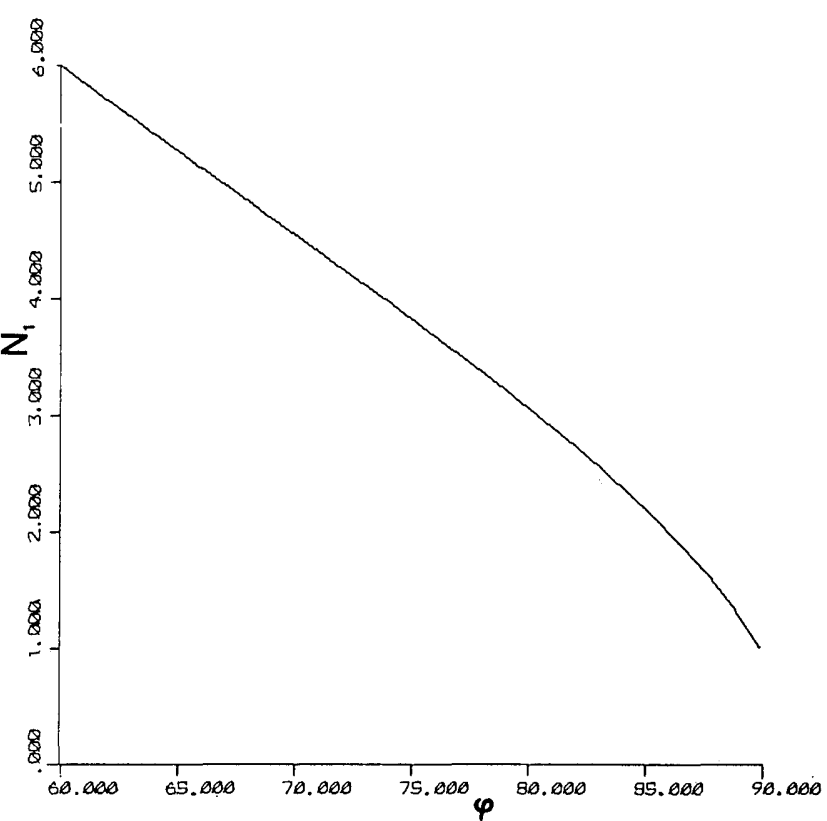

Fig. 4. Refractive index $N_{1}$ of $s$-polarization antireflection layer on $\mathrm{Al}\left(N_{2}=1.212-j 6.924\right)$ as a function of angle of incidence $\phi$ (degrees). The range $\phi<60^{\circ}$ is excluded to avoid far from realistic refractive indices. Light $(\lambda=6328 \AA)$ is assumed to be incident from $\operatorname{air}\left(N_{0}=1\right)$.

can be finely tuned within the desired range $1.78 \leq N_{1}$ $\leq 1.96$ depending on its stoichiometry. ${ }^{14}$

For $\phi=45^{\circ}$, the antireflection layer reduces the $s$ reflectance of the Si surface from $46.94 \%$ to 0 and the $p$ reflectance from 22.04 to $3.61 \%$. Thus the residual reflectance of the coated Si for unpolarized incident light is only $1.8 \%$. At $\phi=75^{\circ}, \mathscr{R}_{p}=37.10 \%$ is not sufficiently high to make the film-substrate system an efficient polarizer. (Reflection from bare Si at the pseudoBrewster angle,,$^{15} \phi=75.44^{\circ}$, makes a simpler more efficient polarizer.)

As a second example, we consider $s$-polarization antireflection layers on an $\mathrm{Al}$ substrate with complex refractive index ${ }^{16} N_{2}=1.212-j 6.924$ at $\lambda=6328 \AA$ in air $\left(\epsilon_{0}=1\right)$. As $\phi$ is increased from 0 to $90^{\circ}, N_{1}$, computed from Eqs. (16)-(18), decreases from 15.07821 to 1 monotonically. To exclude far from realistic values of the film refractive index, $N_{1}(\phi)$ is plotted in Fig. 4 vs $\phi$ over the restricted range $60^{\circ} \leq \phi<90^{\circ}$. The corresponding required normalized $\zeta_{s}$ and actual $d_{s}$ film thicknesses are plotted in Fig. 5 . Notice that $\zeta_{s}$ differs appreciably from $1 / 2$, so that the layer thickness is no longer close to quarterwave. The unextinguished reflectance $\mathscr{R}_{p}$ of the coated surface and the reflectances $\bar{R}_{p}$ and $\overline{\mathscr{R}}_{s}$ of the bare $\mathrm{Al}$ substrate are shown in Fig. 6 as functions of $\phi$. At $A$, Abelès condition is satisfied, as before.

To cite a specific numerical result, we give the characteristics of a thin film on Al that suppresses the reflection of the $s$ polarization at $85^{\circ}$. The required refractive index of the film is $N_{1}=2.220$ (rounded to three decimal places) corresponding to $\mathrm{ZnS}$, for example. ${ }^{12}$ The normalized and actual film thicknesses are $\zeta_{s}=$ 


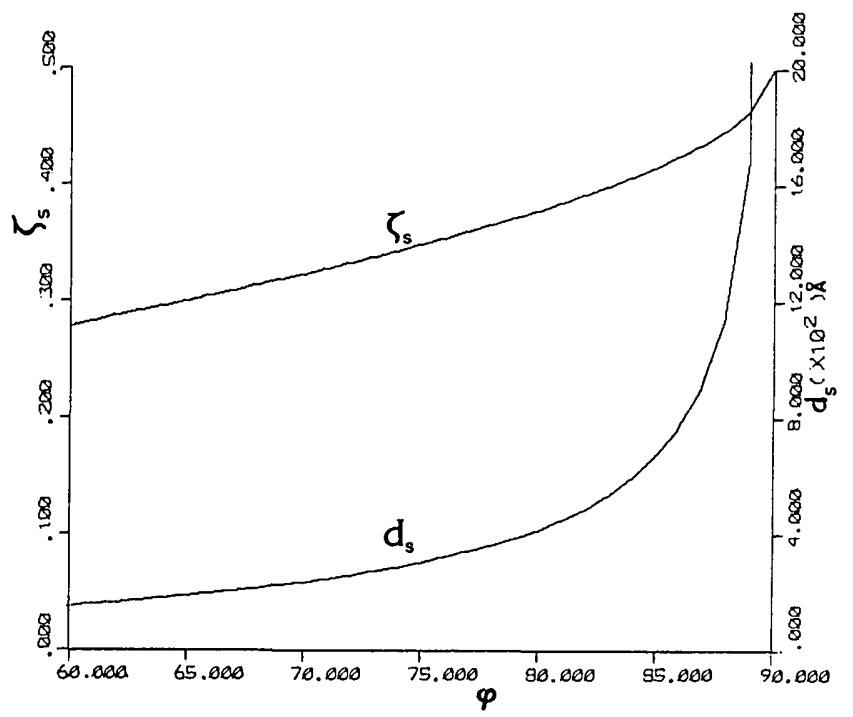

Fig. 5. Normalized $\zeta_{s}$ and actual $d_{s}$ (angstroms) thicknesses of the $s$-polarization antireflection layer on $\mathrm{Al}$ at $\lambda=6328 \AA$ vs angle of incidence $\phi$ (degrees).

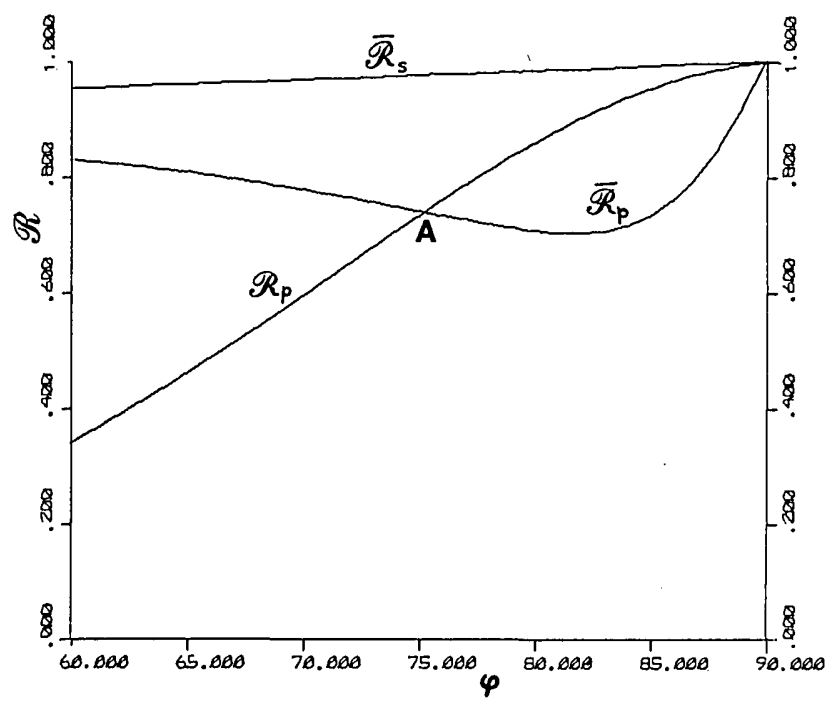

Fig. 6. Unextinguished $p$ reflectance $\mathcal{R}_{p}$ vs angle of incidence $\phi$ (degrees) for Al, which is antireflection-coated for the $s$ polarization. $\overline{\mathcal{R}}_{p}$ and $\overline{\mathcal{R}}_{s}$ are the $p$ and $s$ reflectances of the bare Al substrate.

0.41425 and $d_{s}=661 \AA$. Such a layer reduces the reflectance of $\mathrm{Al}$ for the $s$ polarization from the baresurface value of $99.17 \%$ to zero, while enhancing its $p$ reflectance from 73.03 to $95.26 \%$. Thus this film-substrate system functions as an excellent reflection polarizer.

\section{Antireflection of the $p$ Polarization}

Fresnel's reflection coefficients of the ambient-film and film-substrate interfaces for the $p$ polarization are given by ${ }^{9}$

$$
\begin{aligned}
& r_{01 p}=\left(\epsilon_{1} S_{0}-\epsilon_{0} S_{1}\right) /\left(\epsilon_{1} S_{0}+\epsilon_{0} S_{1}\right), \\
& r_{12 p}=\left(\epsilon_{2} S_{1}-\epsilon_{1} S_{2}\right) /\left(\epsilon_{2} S_{1}+\epsilon_{1} S_{2}\right),
\end{aligned}
$$

where $\epsilon_{i}$ and $S_{i}$ are the same as previously defined in Eqs. (12) and (13). Substitution of Eqs. (19) into Eq.
(4) puts the antireflection condition for the $p$ polarization in the form.

$$
\left(\epsilon_{1}^{2} S_{0}^{2}+\epsilon_{0}^{2} S_{1}^{2}\right) \operatorname{Re}\left(\epsilon_{2}^{*} S_{2}\right)=S_{0}\left(\left|\epsilon_{2}\right|^{2} S_{1}^{2}+\epsilon_{1}^{2}\left|S_{2}\right|^{2}\right) .
$$

By replacing $S_{i}$ from Eq. (12) into Eq. (20) and rearranging, a quadratic equation

$$
A \bar{\epsilon}_{1}^{2}+B \bar{\epsilon}_{1}+C=0
$$

is obtained, where

$$
\begin{aligned}
& A=\cos ^{2} \phi \operatorname{Re}\left[\bar{\epsilon}_{2}^{*}\left(\bar{\epsilon}_{2}-\sin ^{2} \phi\right)^{1 / 2}\right]-\cos \phi\left|\bar{\epsilon}_{2}-\sin ^{2} \phi\right|, \\
& B=\operatorname{Re}\left[\bar{\epsilon}_{2}^{*}\left(\bar{\epsilon}_{2}-\sin ^{2} \phi\right)^{1 / 2}\right]-\cos \phi\left|\bar{\epsilon}_{2}\right|^{2}, \\
& C=-\left(\sin ^{2} \phi\right) B ; \\
& \bar{\epsilon}_{1}=\epsilon_{1} / \epsilon_{0}, \bar{\epsilon}_{2}=\epsilon_{2} / \epsilon_{0}
\end{aligned}
$$

are normalized film and substrate dielectric constants, respectively. Solving Eq. (21) gives

$$
\epsilon_{1}=\epsilon_{0}\left[-B \pm\left(B^{2}-4 A C\right)^{1 / 2}\right] / 2 A,
$$

from which

$$
N_{1}=\epsilon_{1}^{1 / 2} .
$$

Equation (18) has been repeated as Eq. (25) for ease of reference.

Equations (21)-(25) give the desired refractive index of the $p$-polarization single-layer antireflection coating in terms of the ambient dielectric constant $\epsilon_{0}$, substrate complex dielectric constant $\epsilon_{2}=\left(n_{2}-j k_{2}\right)^{2}$, and angle of incidence $\phi$. In the special case of a transparent substrate, $\epsilon_{2}$ is real, and the result reduces to that given by Knittl. ${ }^{3}$ Two values of $N_{1}$ are possible that correspond to the two roots of the quadratic equation. They will be denoted by the additional subscripts + and - , according to the + and - signs that appear in Eq. (24). Because $N_{1}$ must be real and positive, the following conditions must be satisfied:

$$
\begin{aligned}
B^{2}-4 A C & \geq 0, \\
\epsilon_{1} & >0 .
\end{aligned}
$$

We now consider examples of $p$-polarization antireflection layers on the same semiconducting ( $\mathrm{Si})$ and metallic (Al) substrates (at $\lambda=6328 \AA$ and in air) as in Sec. III.

Figure 7 shows $N_{1+}$ and $N_{1-}$ plotted vs $\phi$ between $\phi$ $=0$ and $\phi=90^{\circ}$ for $\mathrm{Si}$. Notice the crossover between the two solutions that takes place as $\phi$ passes through the pseudo-Brewster angle, ${ }^{17} \phi_{\mathrm{pB}}=75.44^{\circ}$, of $\mathrm{Si}$. If the two solutions of $N_{1}$ are ordered according to their magnitudes as low and high, $N_{1 l}$ and $N_{1 h}$, we find that $N_{1 l}<1$ for $\phi<\phi_{\mathrm{pB}}$ and that $N_{1 l}$ is very slightly $>1$ for $\phi>\phi_{\mathrm{pB}}+0.1^{\circ}$. This low-index branch does not correspond to practical thin-film coating materials and will not be pursued further.

Figure 8 shows the normalized and actual film thicknesses $\zeta_{p}\left(=\zeta_{p+}=\zeta_{p-}\right)$ and $d_{p}$, respectively, of the $p$-polarization antireflection layer on $\mathrm{Si} ; d_{p}$ is associated with and calculated from $N_{1 h}$, the higher of the two refractive indices $N_{1+}$ and $N_{1-}$. Significant deviation of the thickness from a quarterwave occurs in the vicinity of the pseudo-Brewster angle. ${ }^{17}$ 


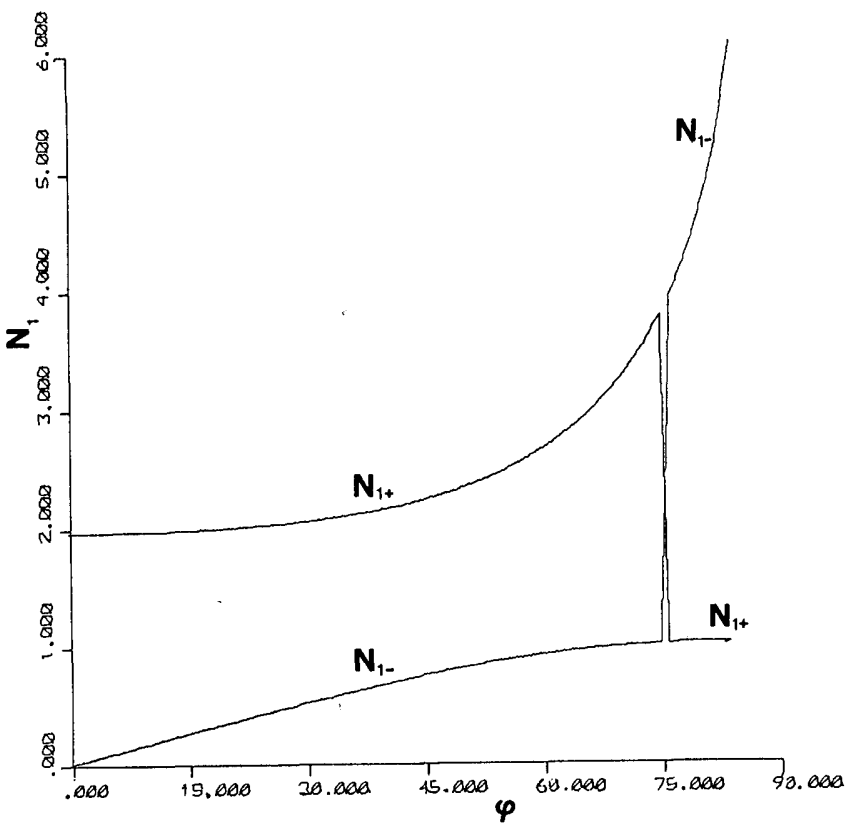

Fig. 7. Refractive indices $N_{1+}$ and $N_{1-}$ of $p$-polarization antireflection layers on $\mathrm{Si}\left(N_{2}=3.85-j 0.02\right)$ as functions of angle of incidence $\phi$ (degrees). Light $(\lambda=6328 \AA)$ is assumed to be incident from $\operatorname{air}\left(N_{0}=1\right)$.

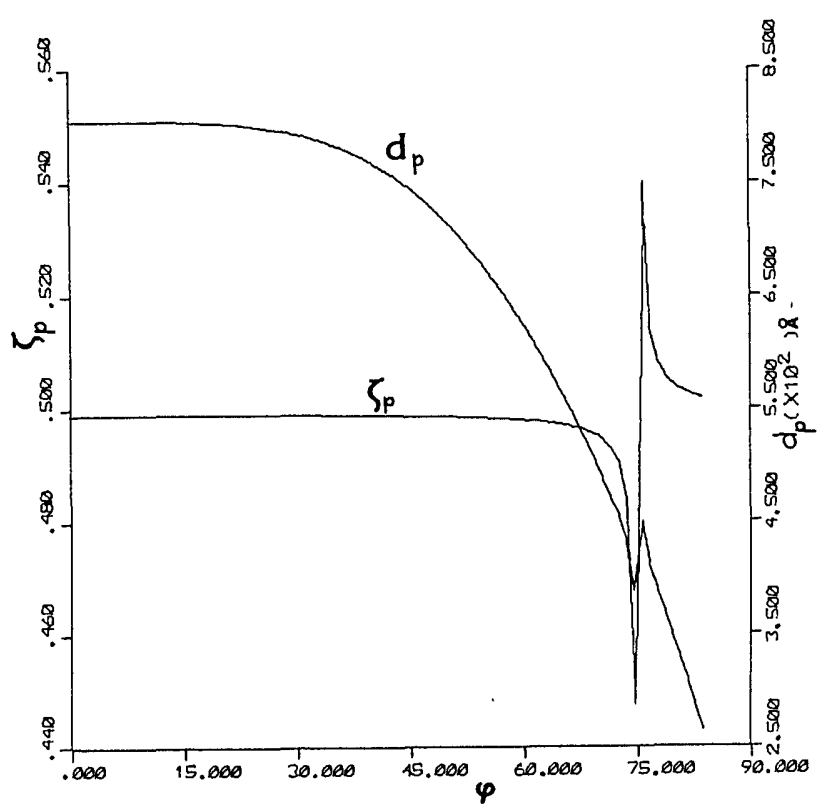

Fig. 8. Normalized $\left(\zeta_{p+}=\zeta_{p-}=\zeta_{p}\right)$ and actual ( $d_{p}$, angstroms) thicknesses of the $p$-polarization antireflection layer on $\mathrm{Si}$ at $\lambda=6328$ $\AA$ vs angle of incidence $\phi$ (degrees). $d_{p}$ corresponds to the higher of the two refractive indices $N_{1+}$ and $N_{1-}$ of Fig. 7 .

Figure 9 shows the unextinguished reflectance $\mathcal{R}_{s}$ of Si coated with the $p$-antireflection layer of index $N_{1 h}$. The bare -Si reflectances $\overline{\mathscr{R}}_{p}$ and $\overline{\mathscr{R}}_{s}$ for the $p$ and $s$ polarizations are also indicated as functions of $\phi$.

Table II summarizes data on $p$-antireflection layers on Si at $\lambda=6328 \AA$ for the same five angles of incidence $\phi=0,30,45,60$, and $75^{\circ}$ considered in Table I. Only the high-index solution is included. The first lines of Tables I and II (at $\phi=0$ ) are identical, as expected.

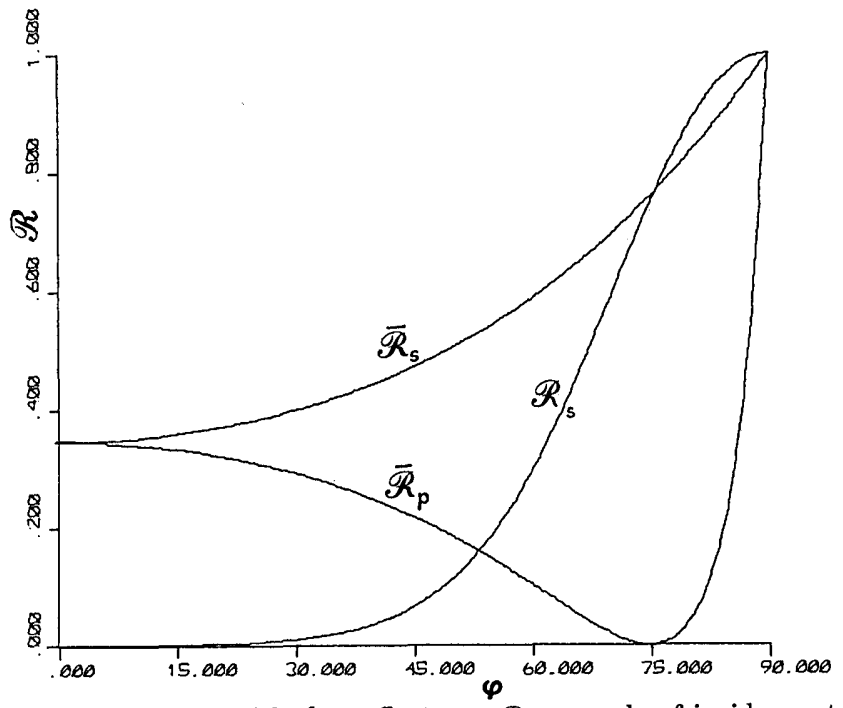

Fig. 9. Unextinguished $s$ reflectance $\mathcal{R}_{s}$ vs angle of incidence $\phi$ (degrees) of $\mathrm{Si}$, which is antireflection-coated for the $p$ polarization. The higher of the two refractive indices $N_{1+}$ and $N_{1-}$ of Fig. 7 is assumed. $\overline{\mathscr{R}}_{p}$ and $\bar{R}_{s}$ are the $p$ and $s$ reflectances of the bare $\mathrm{Si}$ substrate.

Table II. p-Polarization Antireflection Layers on Si at Five Angles of Incidence ${ }^{a}$

\begin{tabular}{lcccl}
\hline$\phi$ & $N_{1}$ & $\zeta_{p}$ & $\mathrm{~d}_{p}$ & \multicolumn{1}{c}{$\mathcal{R}_{s}$} \\
\hline 0 & 1.96218 & 0.49886 & 804.4 & 0 \\
30 & 2.05377 & 0.49874 & 792.2 & 0.0083 \\
45 & 2.23235 & 0.49850 & 744.9 & 0.0637 \\
60 & 2.65778 & 0.49774 & 626.8 & 0.2937 \\
75 & 3.79230 & 0.44725 & 385.9 & 0.7500 \\
\hline
\end{tabular}

${ }^{a} \phi$ is the angle of incidence in degrees. $N_{1}$ is the higher of the two possible refractive indices of the $p$-polarization antireflection layer. $\zeta_{p}$ and $d_{p}$ are the normalized and actual (angstroms) layer thicknesses, respectively. $\mathscr{R}_{s}$ is the unextinguished $s$ reflectance of the $p$-antireflection-coated $\mathrm{Si}$. The complex refractive index of $\mathrm{Si}$ is taken as $N_{2}=3.85-j 0.02$ at $\lambda=6328 \AA$. The reflectances $\overline{\mathcal{R}}_{p}$ and $\overline{\mathcal{R}}_{s}$ of the bare Si substrate are given in Table $\mathrm{I}$.

For overall antireflection at $45^{\circ}, s$ suppression is preferred over $p$ suppression because of the lower associated residual unextinguished reflectance. (At $\phi=$ $45^{\circ}, \mathcal{R}_{p}=3.6 \%$, while $\mathscr{R}_{s}=6.4 \%$ from Tables I and II.)

For the $\mathrm{Al}$ substrate, as $\phi$ is increased from 0 to $34^{\circ}$, Eqs. (21)-(25) yield $N_{1+}$ that increases from 15.07822 to 77.51710 and $N_{1}$ - that increases from $\simeq 0$ to 0.55922 monotonically. These refractive indices are obviously mathematically but not physically acceptable for a single thin film. Between $\phi \simeq 35^{\circ}$ and $\phi=88^{\circ}$, no solutions exist because one or the other of Eqs. (26) is not satisfied. Solutions begin to reappear at $\phi \simeq 88.67^{\circ}$, and realistic refractive indices are obtained only over the very narrow interval from 88.67 to $89^{\circ} . N_{1+}$ and $N_{1-}$ are plotted vs $\phi$ over this range in Fig. 10 . The two solutions merge, $N_{1+}=N_{1-} \simeq \sqrt{ } 2$, at a point $Q$ that corresponds to an angle $\phi_{Q}$ between 88.66 and $88.67^{\circ}$. (At $\phi_{Q}$ light is refracted in the film at $45^{\circ}$, and the $s$ polarization is also suppressed at the same film index but at a different film thickness. ${ }^{18}$ )

The normalized film thicknesses $\zeta_{p+}$ and $\zeta_{p-}$, associated with $N_{1+}$ and $N_{1-}$, respectively, are equal $\left(\zeta_{p+}\right.$ 


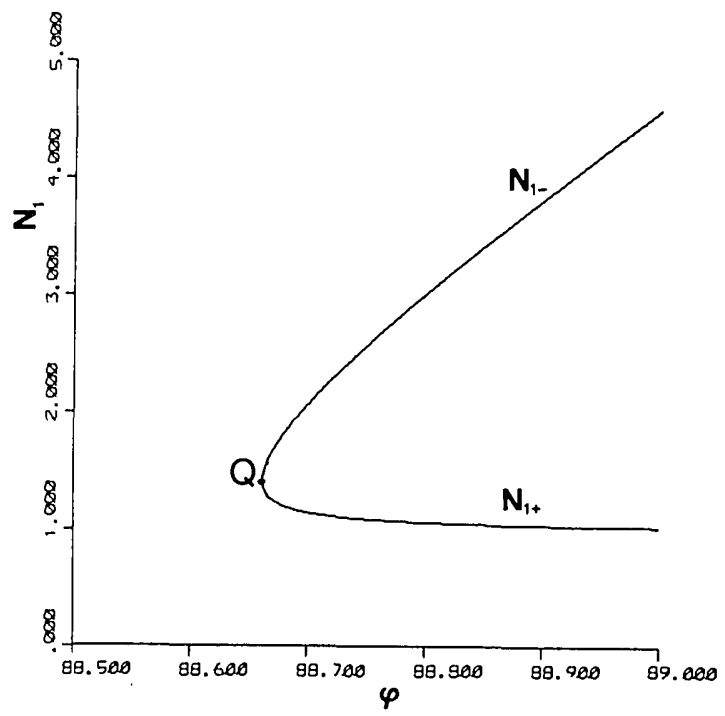

Fig. 10. Refractive indices $N_{1+}$ and $N_{1-}$ of $p$-polarization antireflection layers on $\mathrm{Al}\left(N_{2}=1.212-j 6.924\right)$ as functions of the angle of incidence $\phi$ over the very narrow range $88.5^{\circ} \leq \phi \leq 89^{\circ}$. No solutions for $N_{1}$ exist, or far from practical values are obtained, outside this range of $\phi$. The two solutions $N_{1+}$ and $N_{1-}$ merge at point $Q$. Light $(\lambda=6328 \AA)$ is assumed to be incident from $\operatorname{air}\left(N_{0}=1\right)$.

$=\zeta_{p-}=\zeta_{p}$ ) and decrease monotonically from 0.908415 to 0.811834 as $\phi$ increases from 88.67 to $89^{\circ}$. The unextinguished reflectance $\mathcal{R}_{s+}$ decreases very slightly from 99.774 to $99.769 \%$, while $\mathcal{R}_{s-}$ increases little from 99.784 to $99.880 \%$ monotonically over the same range of $\phi$. Except that the angle of incidence is too close to $90^{\circ}$, this film-substrate system acts as a nearly ideal reflection polarizer.

\section{Summary}

For any given interface between (linear, nonmagnetic, and optically isotropic) transparent and absorbing media, the refractive index $N_{1}$ of an intermediate transparent layer can be found that allows suppression of the reflection of $p$-or $s$-polarized light at a specified angle of incidence $\phi$. The explicit equations that determine $N_{1}$ are Eqs. (16)-(18) for the $s$ polarization, and Eqs. (21)-(25) for the $p$ polarization. $N_{1}$ must correspond to the refractive index of an existing thin-film coating material for the mathematical solution to be physically realizable by a single film. Thus $p$ and $s$ antireflection will often be possible only over limited ranges of $\phi$, depending on the substrate and ambient optical constants. The thickness of the antireflection layer and the reflectance of the coated substrate for the unextinguished orthogonal polarization are calculated, also explicitly, from Eqs. (5)-(8) and Eqs. (9) and (10), respectively. The method is applied to $\mathrm{Si}$ and $\mathrm{Al}$ substrates at $\lambda=6328 \AA$, and the results appear graphically and in tables.

I am pleased to acknowledge the support received from the State of Louisiana Board of Regents and the Foundation for A Better Louisiana and the assistance of Karim Javily in generating the numbers and figures.

\section{References}

1. M. Born and E. Wolf, Principles of Optics (Pergamon, New York, 1975), p. 64.

2. J. Strong, "On a Method of Decreasing the Reflection from Nonmetallic Substances," J. Opt. Soc. Am. 26, 73 (1936).

3. Z. Knittl, Optics of Thin Films (Wiley, New York, 1976), p. 73.

4. G. Hass, H. H. Schroeder, and A. F. Turner, "Mirror Coatings for Low Visible and High Infrared Reflectance," J. Opt. Soc. Am. 46, 31 (1956); K. C. Park, "The Extreme Values of Reflectivity and the Conditions for Zero Reflection from Thin Dielectric Film on Metal," Appl. Opt. 3, 877 (1964).

5. M. Ruiz-Urbieta and E. M. Sparrow, "Reflection Polarization by a Transparent-Film-Absorbing Substrate System," J. Opt. Soc. Am. 62, 1188 (1972).

6. R. M. A. Azzam, A.-R. M. Zaghloul, and N. M. Bashara, "Ellipsometric Function of a Film-Substrate System: Applications to the Design of Reflection-Type Optical Devices and to Ellipsometry," J. Opt. Soc. Am. 65, 252 (1975).

7. H. Kitajima, K. Fujita, and H. Cizmic, "Zero Reflection from a Dielectric Film on Metal Substrate at Oblique Angles of Incidence," Appl. Opt. 23, 1937 (1984).

8. For given optical constants of the ambient, film, and substrate, Eq. (3) becomes a transcendental equation in the angle of incidence $\phi$ that can be solved by iteration. ${ }^{5-7}$ For high-reflectance (metal) substrates, explicit equations for the polarizing angles, that are approximate but accurate, can be obtained.

9. See, for example, R. M. A. Azzam and N. M. Bashara, Ellipsometry and Polarized Light (North-Holland, Amsterdam, 1977), Chap. 4.

10. G. Gergely, Ed., Ellipsometric Tables of the $\mathrm{Si}_{-} \mathrm{SiO}_{2}$ System for Mercury and He-Ne Laser Spectral Lines (Akademiai Kiado, Budapest, 1971).

11. E. Ritter, "Optical Film Materials and Their Applications," Appl. Opt. 15, 2318 (1976).

12. H. K. Pulker, "Characterization of Optical Thin Films," Appl. Opt. 18, 1969 (1979).

13. O. S. Heavens, Optical Properties of Thin Solid Films (Dover, New York, 1965), p. 119

14. G. Eisenstein and L. W. Stulz, "High Quality Antireflection Coatings on Laser Facets by Sputtered Silicon Nitride," Appl. Opt. 23, 161 (1984).

15. R. M. A. Azzam and T. F. Thonn, "Pseudo-Brewster and Second-Brewster Angles of an Absorbing Substrate Coated by a Transparent Thin Film," Appl. Opt. 22, 4155 (1983).

16. T. H. Allen, "Study of Al with Combined Auger SpectrometerEllipsometer System," J. Vac. Sci. Technol. 13, 112 (1976).

17. Examining the behavior of the solutions in the immediate neighborhood (within $\pm 0.01^{\circ}$ ) of the pseudo-Brewster angle $\phi_{\mathrm{pB}}$ reveals some interesting detail. For example, there is a gap in this already very narrow range of $\phi$ where no solutions exist. The gap is approximately bounded by the angles $\phi_{1}=75.4397^{\circ}$ and $\phi_{2}=75.4401^{\circ}$ and includes $\phi_{\mathrm{pB}}$. The solutions $N_{1+}$ and $N_{1-}$ merge at the upper edge of the gap to form one continuous curve. Furthermore, the concident $\zeta_{p+}$ and $\zeta_{p-\text { tend to zero as the lower }}$ gap edge is approached from the left and tend to one as the upper gap edge is approached from the right.

18. Elsewhere we consider directly and in detail the conditions for the extinction of the $p$ and $s$ polarizations at the same angle of incidence by a transparent film on an absorbing substrate along with interesting applications; see R. M. A. Azzam, "Extinction of the $p$ and $s$ Polarizations of a Wave on Reflection at the Same Angle from a Transparent Film on an Absorbing Substrate: Applications to Parallel-Mirror Crossed Polarizers and a Novel Integrated Polarimeter," J. Opt. Soc. Am. A 2, in press (1985). 